\title{
A REVIEW PAPER ON CLOUD COMPUTER ARCHITECTURE
}

\author{
Dr. Kashif Qureshi \\ SOEIT, Sanskriti University, Mathura, Uttar Pradesh, India
}

\begin{tabular}{|c|c|c|c|}
\hline Journal & \multicolumn{3}{|c|}{$\begin{array}{l}\text { Samvakti Journal of Research in Information Technology } \\
\text { https://www.sjrit.samvaktijournals.com } \\
\text { Volume } 2 \text { Year of Volume } 2021 \text { Page No : } 16-23\end{array}$} \\
\hline Discipline & \multicolumn{3}{|l|}{ Cloud Computing } \\
\hline Conference & \multicolumn{3}{|c|}{$\begin{array}{l}\text { A virtual international conference on redefining and transforming the role of higher } \\
\text { education in sustainable development }\end{array}$} \\
\hline Conference & \multicolumn{3}{|c|}{ Start Date: September 30, 2021} \\
\hline Dates & \multicolumn{3}{|c|}{ End Date : September 30, 2021} \\
\hline Institute Name & \multicolumn{3}{|c|}{$\begin{array}{l}\text { JAIN (Deemed-to-be University) in association with Council for Industrial } \\
\text { Innovation and Research }\end{array}$} \\
\hline $\begin{array}{l}\text { Date Received } \\
\text { ID } \\
\text { Dol }\end{array}$ & $\begin{array}{l}\text { November 19, } 2021 \\
2021.02 .12 \\
10.46402 / 2021.02 .12\end{array}$ & $\begin{array}{l}\text { Publication Date } \\
\text { Paper Type } \\
\text { Dol URL }\end{array}$ & $\begin{array}{l}\text { : December 11, } 2021 \\
\text { : Conference Paper } \\
\text { : https://dx.doi.org/10.46402/2021.02.12 }\end{array}$ \\
\hline
\end{tabular}

\section{ABSTRACT}

Due to significant developments in processing and memory technology, as well as the number of web Users, computers are becoming less, better potent, but more generally available than it has ever been. As a consequence of this technical movement, cloud technology has emerged as a revolutionary cloud architecture in which capabilities are supplied as public commodities that customers may purchase and discharge on it over the Network. Data centres, who run online services and license available as a supplement about use, and wireless carriers, who purchase capabilities through one or more infrastructures to support consumers, are the two types of services suppliers in a virtualized system. In recent years, Web desktop technology seems to have had a substantial effect on the Search Engine Company, with giant conglomerates such as Google, Amazon, and Microsoft attempting to create more important, reliable, and expense virtualisation mineral wealth, and businesses merely reorganizing their promotional methodologies to take account of new concept. Public cloud, as seen below, offers a variety of attractive properties that makes it interesting to business leaders.

KEYWORDS: Cloud Computing, laaS, Information, PaaS, SaaS, Technology. 


\section{INTRODUCTION}

There are almost no out-of-pocket expenses: Pay-as-you-go is the cornerstone of virtualization. A phone company does not want to build equipment to begin reaping the benefits of clouds technology. It only rents \& charges for cloud services depending by its own usage. Decreased operating costs: Instruments may well be swiftly allotted and de-allocated in a virtual system. As a consequence, a free company is no immediately required to offer resources in response to maximum demands. This saves a lot of money since resources may be freed to reduce operational expenses when demand is low. High scalability: Infrastructure providers combine a huge number of data center resources and make them readily available. In order to meet fast increases in service demand, a service provider may simply extend its service to enormous sizes (e.g., flash-crowd effect) ${ }^{[1][2][3]}$.

1. Easy access: Cloud-based services are often web-based. As a result, they may be accessed from a wide range of Internet-connected devices. These gadgets include not just desktop and laptop computers, but also mobile phones and personal digital assistants (PDAs).

2. Reduced business risks and maintenance costs: When a service provider outsources its infrastructure to the cloud, it transfers its business risks (such as hardware failures) to infrastructure providers, who typically have more knowledge and are better prepared to manage these risks. Furthermore, a service provider may reduce the expenses of hardware maintenance and employee training.

However, although cloud computing has provided significant benefits to the IT sector, it also poses a number of unique problems that must be properly handled. We provide a review of cloud computing in this article, emphasizing important ideas, architectural principles, cutting-edge implementations, and research difficulties. Our goal is to get a better grasp of cloud computing's design problems and to identify significant research paths in this interesting field.

\section{Cloud Computing:}

Virtualization isn't a brand-new notion. Since then, internet has very much been seen as a promotional term to express a range of ideas in a range of circumstances. The lack of a precise concept of cloud services has surely led in scepticism and confusion, as well as business enthusiasm. As a consequence, substantial attempts to regulate the notion of virtualization have already been launched. For example, in attempting to develop a common description, academics looked at nearly 20 different definitions from diverse resources.

The fundamental aspects of cloud computation ${ }^{[4]}$ :

Information system is defined by the American International standardization Organization. Computation is a technique for providing on-demand connected connectivity to large quantities of programmable computational power that can be 
instantaneously offered and removed with minimum administrative effort or involvement from commercial suppliers.

The main explanation for the gap in virtualization views is that, unlike many other technology terms, clouds storage refers to a new operational approach that combines a variety of current technology to manage a business in a new way. Virtualisation and convenience charging, for example, aren't novel cloud processing technology. Cloud storage, but at the other hand, employs contemporary capabilities ${ }^{[5][6][7]}$.

\section{Technologies:}

Cloud technology often is contrasted to the systems mentioned beneath, because all hold some of the same properties as virtualization:

\section{a. Grid computing:}

Array technology is a decentralized cloud processing model that coordinates connected capabilities to achieve a common mathematical objective. Server virtualization was spurred by engineering coding, which are frequently computationally expensive. Virtualization and Spread systems are similar in that they always rely on dispersed opportunities to ensure implementation objectives. Cloud technology, and from the other hand, pushes went a step forward by enabling data exchange and adaptive supply allocation via the use of computer science systems at various levels.

\section{b. Utility computing:}

Network virtualization is the notion of providing on-demand capabilities to clients and compensating them based on use or perhaps a set charge. Cloud storage might be considered something of a server virtualization. Only for monetary reasons does it adopt an energy pricing structure. Using it on capacity allocation and energy payment, network operators may really maximize resources utilization and save operating expenses.

\section{c. Virtualization:}

Virtualisation is a method of abstracting additional equipment details from slightly elevated programs and providing virtualisation technology. A virtualization is a word used to characterize a system that has been automated (VM). Virtual box is fundamental to public cloud because it enables the aggregation of computational power from several machines as well as the variable distribution and redeployment of virtualisation to programs on required.

\section{Cloud Computing Architecture:}

This section discusses cloud computing's architectural, business, and operational paradigms. 


\section{a. A cloud computing layered model:}

The architectural of a virtualized system may be divided into four layers: equipment layer, network level, platforms layer, and application server. We go and via this one in depth:

\section{i. Hardware Layer:}

The hardware layer consists of:

The spare parts of the internet, including such real computers, networks, exchanges, electricity, and coolant equipment, are managed by this level. In practice, the equipment layer is created in network infrastructure. In a datacentre, hundreds of computers are generally organized in cabinets and connected with switching, gateways, and perhaps other textiles. At the hypervisor level, frequent issues include modular architecture, flexibility, transport control, and electricity and conditioning micromanagement.

\section{ii. Infrastructure Layer:}

The cloud infrastructures, also referred as the digital machine, creates a pooling of huge computational capabilities by partitioning material capabilities using virtualisation such as KVM, Xen, and VMware. Because several critical characteristics, including energy allocation and redistribution, are only accessible through programming languages, the networking application is an important form of web technology.

\section{iii. Platform Layer}

Software packages and system architectures make up the platforms layers, which lies on highest point of the network level. The foundation layer's purpose is to making it as easy as practicable to deploy applications directly into VM environments. At the android foundation, Google App Engine, for example, offers API functionality for building memory, databases, and system behavior in traditional software programs.

\section{iv. Application Layer}

The genuine internet programs especially at the server side, and it's at the head of the pyramid. With traditional programmes, internet applications might taking advantage of automated scalability to increase productivity, reliability, and operating expenses. Unlike traditional services providing environments such as standalone network infrastructure, cloud gaming infrastructure seems to be more adaptable. Each level is only loosely linked to those over and underneath, allowing it to evolve freely. This is similar to whatever the OSI internetwork paradigm is built. Cloud computing's architectural flexibility enables it to accommodate a broad variety of application needs while lowering administration and maintenance costs. Figure 1 shows the Cloud Computing Architecture. 


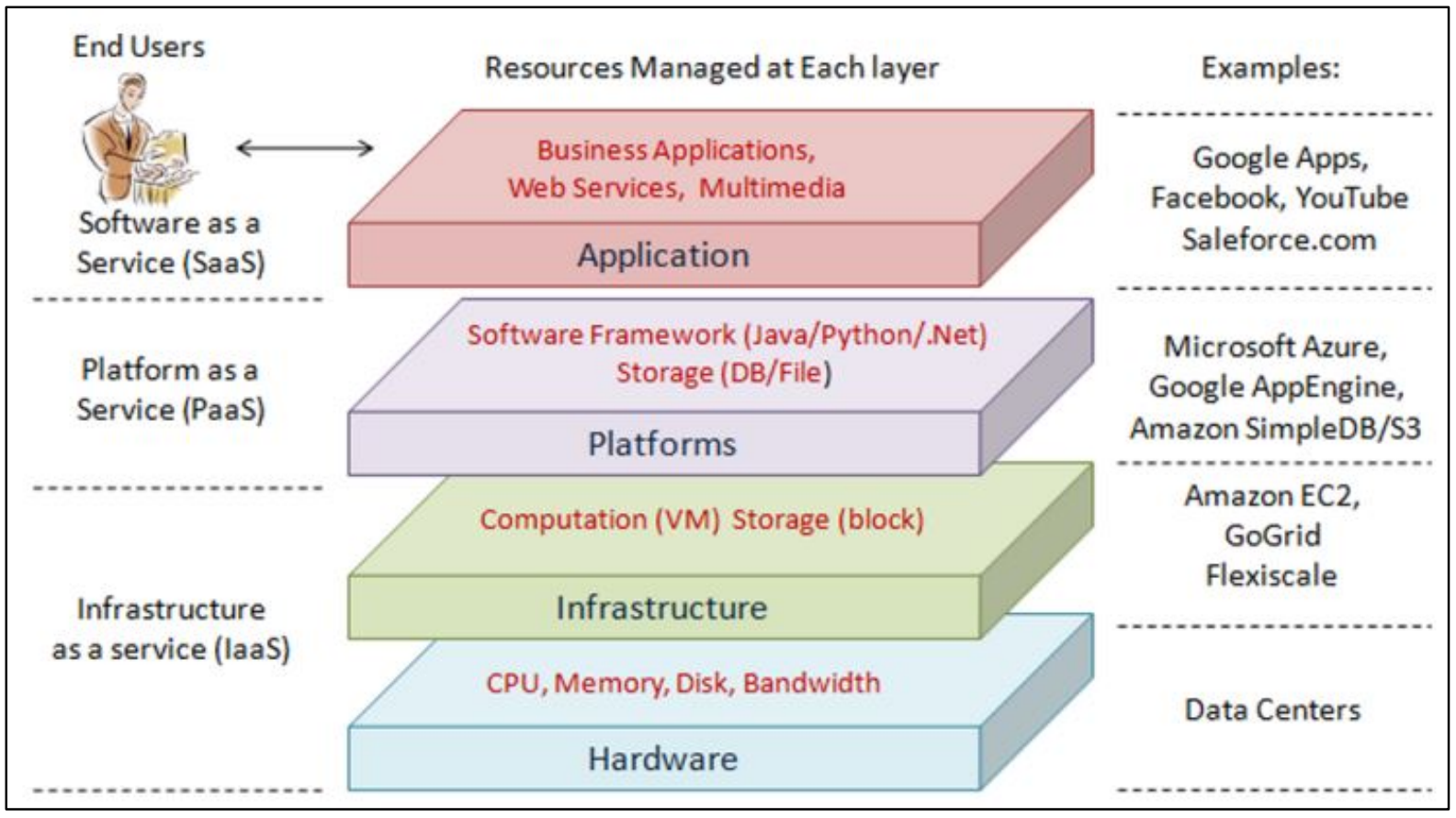

Figure 1: The above figure shows the Cloud Computing Architecture.

\section{Business Strategy}

Virtualization has a service-oriented corporate structure. Infrastructure and marketplace components are rendered accessible as it is on operations, to put it differently ways. From a logical approach, each level of the design mentioned in the previous paragraph may be performed as a services to the element above. That each layers, but at the opposite side, may be considered a customer of the layer behind it. Cloud, on the other hand, offer three sorts of facilities:

\section{a. Software as a service (SaaS):}

The term "cloud computing model" relates to the delivery of construction provisioning, usually in the shape of simulated computers (VMs).

b. Platform as a service (PaaS):

The providing of platforms level elements also including runtime environment assistance and project management environments is referred to as PaaS (Platform as a Service).

\section{c. Infrastructure as a service (laaS):}

SaaS is a term that describes how information is distributed through the Internet. laaS is a term that refers to the provision of technology resources through the Internet. 


\section{Types of Cloud:}

There are several factors to examine before transferring a business system to the internet. Several wireless carriers, for instance, seem mainly preoccupied with cutting prices, when others place a premium on reliability and protection.

\section{a. Public Cloud:}

In a cloud infrastructure, telecommunications companies makes various capabilities accessible as a commodity to the general population. For service businesses, cloud servers offer several benefits, along with no initial investments in technology and risk sharing to data centers. The absence of quite ok command on communication, networking, and access controls in virtualized environments, but from the opposite side, restricts their applicability in several business applications.

\section{b. Private Cloud:}

Private clouds, often referred as personal data center computing, are meant for the unique use of a particular company. A corporate network could be built and managed throughout or with the help of third technologies. In terms of speed, reliability, and confidentiality, a cloud hosting offers the greatest freedom. They are, nonetheless, often lambasted for resembling traditional commercial datacenters and neglecting continue providing benefits as in no forward investment costs.

\section{c. Hybrid Clouds:}

A clouds service combines cloud - based architectures to address the shortcomings of every. In a virtual server, some of the network application runs on virtualized solutions, while its remainder works on cloud services. The versatility of cloud services is lacking both in virtualized applications. They give greater management and safety around database objects than virtual disk systems while also enabling on-demand services development and limitation. On the downside, constructing a clouds platform necessitates painstakingly determining the best range of formal and informal server implementations.

\section{DISCUSSION}

The author has discussed about the cloud computing architecture, reduced operating costs: Resources may be quickly assigned and re-allocated in a cloud environment. Therefore, there is no need of service provider to supply capacity in response to peak demand. When demand is low, resources may be freed up to decrease operating costs, which saves a lot of money. The rapid advances in processing and storage technology, as well as the rise of online, cloud servers have become faster, more sophisticated, and more widely available than ever before. Virtualization has arisen as a digital operating architecture as a result of this technological development, in which facilities (such as CPU and storage) are given as universal utility that consumers might 
buy and distribute over the World Wide Web. In an internet surroundings, there are two forms of business providers: construction server supplier who handle computing solutions and lease mineral wealth based on usage, and telephone corporations who rent potency from one maybe more wireless carriers to function recipients.

\section{CONCLUSION}

Public cloud is a fascinating framework for organizing and providing facilities that has just emerged in recent years, as the authors has highlighted. Public cloud is rapidly changing the world of digital technologies, delivering utilitarian computing's longawaited promised further to fruition. Despite all the benefits of cloud technology, current techniques are not yet sophisticated sufficiently here to fully realize its promise. Many essential issues in this field, such as automatic service selection, electricity efficiency, and information privacy, are now just being studied in academia. As a result, we think there is still a lot of room for researchers to make major discoveries in this area and have a big effect on the industry's growth. 


\section{REFERENCES}

[1] I. A. T. Hashem, I. Yaqoob, N. B. Anuar, S. Mokhtar, A. Gani, and S. Ullah Khan, "The rise of 'big data' on cloud computing: Review and open research issues," Information Systems. 2015, doi: 10.1016/j.is.2014.07.006.

[2] V. Pushpalatha, K. B. Sudeepa, and H. N. Mahendra, "A survey on security issues in cloud computing," Int. J. Eng. Technol., 2018, doi: 10.23956/ijarcsse/sv7i5/0217.

[3] K. Akherfi, M. Gerndt, and H. Harroud, "Mobile cloud computing for computation offloading: Issues and challenges," Applied Computing and Informatics. 2018, doi: 10.1016/j.aci.2016.11.002.

[4] C. Stergiou, K. E. Psannis, B. G. Kim, and B. Gupta, "Secure integration of loT and Cloud Computing," Futur. Gener. Comput. Syst., 2018, doi: 10.1016/j.future.2016.11.031.

[5] B. de Bruin and L. Floridi, "The Ethics of Cloud Computing," Sci. Eng. Ethics, 2017, doi: 10.1007/s11948-016-9759-0.

[6] Q. Zhang, L. Cheng, and R. Boutaba, "Cloud computing: State-of-the-art and research challenges," J. Internet Serv. Appl., 2010, doi: 10.1007/s13174-0100007-6.

[7] S. Shilpashree, R. R. Patil, and C. Parvathi, "'Cloud computing an overview," Int. J. Eng. Technol., 2018, doi: 10.14419/ijet.v7i4.10904.

\section{$\underline{\underline{E N D}}$}

